Serum non-cholesterol sterols and cholesterol metabolism in childhood and adolescence

\title{
Gylling, Helena
}

2018-11

Gylling , H , Korhonen , M , Mutanen , A , Nissinen , M J , Pakarinen , M \& Simonen , P 2018 , ' Serum non-cholesterol sterols and cholesterol metabolism in childhood and adolescence ' , Atherosclerosis , vol. 278 , pp. 91-96 . https://doi.org/10.1016/j.atherosclerosis.2018.09.017

http://hdl.handle.net/10138/307719

https://doi.org/10.1016/j.atherosclerosis.2018.09.017

publishedVersion

Downloaded from Helda, University of Helsinki institutional repository.

This is an electronic reprint of the original article.

This reprint may differ from the original in pagination and typographic detail.

Please cite the original version. 


\title{
Serum non-cholesterol sterols and cholesterol metabolism in childhood and adolescence
}

\author{
Helena Gylling ${ }^{\mathrm{a}, *}$, Matilda Korhonen ${ }^{\mathrm{b}}$, Annika Mutanen ${ }^{\mathrm{c}}$, Markku J. Nissinen ${ }^{\mathrm{d}}$, Mikko Pakarinen ${ }^{\mathrm{c}}$, \\ Piia Simonen ${ }^{\mathrm{b}}$ \\ a Department of Internal Medicine, University of Helsinki and Helsinki University Hospital, PO BOX 700, 00029 HUS, Helsinki, Finland \\ ${ }^{\mathrm{b}}$ Heart and Lung Center, Department of Cardiology, University of Helsinki and Helsinki University Hospital, PO BOX 340, 00029 HUS, Helsinki, Finland \\ ' Children's Hospital, Pediatric Surgery, University of Helsinki and Helsinki University Hospital, PO BOX 281, 00029 HUS, Helsinki, Finland \\ d Abdominal Center, Department of Gastroenterology, University of Helsinki and Helsinki University Hospital, PO BOX 700, 00029 HUS, Helsinki, Finland
}

\section{H I G H L I G H T S}

- We evaluated cholesterol metabolism in 0-15 year-old children without dyslipidemia.

- Serum non-cholesterol sterols depicted cholesterol synthesis and absorption.

- From the age of 1 year, cholesterol homeostasis was intact.

- From the age of 1-10 years cholesterol absorption prevailed cholesterol synthesis.

- This new finding emphasizes the importance of healthy diet from early childhood.

\section{A R T I C L E I N F O}

\section{Keywords:}

Children

Cholesterol

Cholesterol absorption

Cholesterol synthesis

Desmosterol

Lathosterol

\begin{abstract}
A B S T R A C T
Background and aims: The profile of cholesterol metabolism, i.e., high absorption vs. high synthesis, may have a role in the development of atherosclerosis, the early lesions of which can be present already in childhood. Since there is no information on cholesterol metabolism in children from birth to adolescence, we evaluated cholesterol metabolism in 0-15 year-old children and adolescents without dyslipidemia.

Methods: The study population consisted of 96 children (39 girls, 57 boys) divided into age groups $<1$ ( $n=14$ ), 1-5 $(\mathrm{n}=37), 6-10(\mathrm{n}=24)$, and $11-15(\mathrm{n}=21)$ years. Cholesterol metabolism was assessed by analysing serum non-cholesterol sterols, biomarkers of cholesterol synthesis and absorption, with gas-liquid chromatography.

Results: Serum non-cholesterol sterol ratios to cholesterol did not differ between gender. Cholesterol precursors squalene, cholestenol, and desmosterol were higher in the $<1$ year than in the older age groups, whereas lathosterol was highest in the 11-15 year old. Plant sterols were low in the age group $<1$ year, after which they did not differ between the groups. Cholestanol was not age-dependent. From the age of 1 year, cholesterol homeostasis was intact. Cholesterol absorption prevailed cholesterol synthesis from 1 to 10 years of age (e.g., lathosterol/cholestanol ratio $0.35 \pm 0.03$ and $0.45 \pm 0.05$ in $1-5$ and $6-10$ vs. $0.66 \pm 0.08$ in 11-15 year-old (mean \pm SE, $p<0.001$ ).

Conclusions: Serum non-cholesterol sterols had different individual profiles by age in childhood and adolescence. From 1 to 10 years of age, cholesterol absorption prevailed cholesterol synthesis. This novel finding emphasizes the importance of dietary aspects related to cardiovascular risk even from early childhood.
\end{abstract}

\section{Introduction}

Cholesterol is an essential lipid for the development, growth, and wellbeing of humans. In newborn children, cord blood cholesterol concentration is $1.5 \mathrm{mmol} / \mathrm{l}$, on average [1-4]. During the first year of life, cholesterol concentration in serum increases to about $4-5 \mathrm{mmol} / 1$ [5], a level that remains throughout the childhood [6]. Serum cholesterol concentration, as well as cholesterol metabolism, is regulated by genetic, dietary, hormonal, and lifestyle factors, e.g., in newborns with heterozygous familial hypercholesterolemia, the concentration of cord

\footnotetext{
* Corresponding author. Biomedicum Helsinki C 4 22, P.O. BOX 700, 00029 HUS, Helsinki, Finland.

E-mail address: helena.gylling@hus.fi (H. Gylling).
} 
blood cholesterol is elevated up to about $2.4 \mathrm{mmol} / \mathrm{l}$, and at the age of 1 year, serum cholesterol concentration is about $8 \mathrm{mmol} / 1$ [2]. Already in childhood and adolescence, elevated serum and LDL cholesterol concentration can lead to atherosclerotic changes in arterial wall [7]. In addition to LDL cholesterol, the whole-body cholesterol metabolism may have a role in the development of atherosclerosis. This was suggested in adult cohorts in which high cholesterol absorption efficiency increased the risk of coronary events regardless of LDL cholesterol concentration [8-11]. The growth and development in childhood and adolescence question the whole-body cholesterol metabolism, which may vary between different ages. To our knowledge, there is only a limited number of studies on limited age periods dealing with cholesterol metabolism in healthy children and adolescents [1-4,12-14], and there are no studies reaching all age groups from newborn to adolescence. Thus, there is no information on whether the metabolic profile of cholesterol differs between age groups or gender in children and adolescents. Therefore, the aim of this study was to evaluate cholesterol metabolism in healthy children from 0 to 15 years of age by analysing serum non-cholesterol sterols, which in general are used as biomarkers of cholesterol synthesis and cholesterol absorption [15-19].

\section{Patients and methods}

\subsection{Study population}

The study population consisted of consecutive 0-15 year-old daysurgery patients of our hospital during the years 2009-2015, who were otherwise healthy and did not have dyslipidemia, diabetes, thyroid, renal, hepatobiliary, gastrointestinal, or malignant disease. Written informed consent was received from all patients or their caregivers before any procedure. The study was performed according to the principles of the Declaration of Helsinki. The Ethics Committee of the Helsinki University Central Hospital had approved the study protocol.

\subsection{Study design}

Fasting blood samples were drawn after a 12-h fast in the morning before the surgery. The subjects of the study population were weighed and height was measured, and medical history and possible drug treatment were recorded from the medical records. All subjects had been on their habitual home diet, but more detailed dietary information or the breastfeeding status was not recorded.

\subsection{Methods}

Serum cholesterol and non-cholesterol sterols and squalene were quantified by capillary gas-liquid chromatography (GLC) with flame ionization detection and using a 50-m capillary column (Ultra 2, Agilent Technologies, Wilmington, DE) with $5 \alpha$-cholestane as the internal standard [20]. The following non-cholesterol sterols were analysed: squalene, cholestenol ( $5 \alpha$-cholest-8-en-3 $\beta$-ol), desmosterol, and lathosterol (cholesterol precursors), cholestanol (a metabolite of cholesterol), and campesterol, sitosterol, stigmasterol, and avenasterol (plant sterols). To eliminate the effects of variation of non-cholesterol sterol transporters (mainly LDL) on the serum sterol concentrations, the non-cholesterol sterol concentrations were standardized and divided by the cholesterol concentration of the same GLC run and expressed as ratios to cholesterol $\left(10^{2} \mathrm{x} \mu \mathrm{mol} / \mathrm{mmol}\right.$ of cholesterol). In general, the ratios to cholesterol of the serum cholesterol precursors reflect cholesterol synthesis, while those of plant sterols (campesterol, sitosterol, and avenasterol) and cholestanol reflect cholesterol absorption efficiency. We also calculated the synthesis marker/absorption marker ratios cholestenol/cholestanol and lathosterol/cholestanol, which reflect cholesterol metabolism, the lathosterol/desmosterol ratio reflecting the activity of the two pathways of cholesterol synthesis, and the campesterol/cholestanol ratio, a biomarker of dietary phytosterol intake [21].

\subsection{Statistics}

Statistical analyses were performed with SPSS for Windows 22.0 (SPSS, Chicago, IL). The normality and homogeneity of variance assumptions were checked before further analyses. Comparison of continuous variables between groups were performed by analysis of variance followed by independent samples $t$-test or Mann-Whitney test if the normality and homogeneity of variance were not reached after logarithmic transformation. Non-continuous variables were tested with Chi-square or Fisher's exact test. Spearman correlation coefficients were calculated. To control the overall level, Bonferroni adjustment was used. A $p$-value of $<0.05$ was considered statistically significant. The results are expressed as mean \pm SE.

\section{Results}

The study population consisted of 96 children and adolescents. Thirty-nine subjects were girls and 57 were boys (Table 1 ). The mean age of the study population was $6.6 \pm 0.5$ (SE) years ranging from 0.10 to 15.94 years, and there was no difference between girls and boys.

Table 1

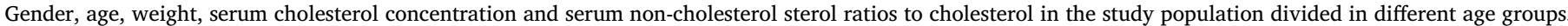

\begin{tabular}{|c|c|c|c|c|c|}
\hline Variables & Age $<1$ year $n=14$ & Age $1-5$ years $n=37$ & Age $6-10$ years $n=24$ & Age $11-15$ years $n=21$ & $p$ \\
\hline Girls/boys, n & $3 / 11$ & $17 / 20$ & $11 / 13$ & $8 / 13$ & 0.670 \\
\hline Age, years & $0.36 \pm 0.06$ & $3.82 \pm 0.22$ & $8.27 \pm 0.29$ & $13.62 \pm 0.34$ & $<0.001$ \\
\hline Weight, kg & $7.6 \pm 1.2$ & $16.5 \pm 0.6$ & $29.7 \pm 1.6$ & $51.1 \pm 2.9$ & $<0.001$ \\
\hline Cholesterol, mmol/1 & $3.38 \pm 0.20$ & $3.75 \pm 0.1$ & $4.14 \pm 0.14^{b}$ & $3.94 \pm 0.14$ & 0.008 \\
\hline Squalene $^{\mathrm{a}}$ & $21.6 \pm 1.9$ & $13.0 \pm 0.9^{\mathrm{b}}$ & $12.7 \pm 0.7^{\mathrm{b}}$ & $16.3 \pm 1.7$ & $<0.001$ \\
\hline Cholestenol $^{\mathrm{a}}$ & $20.7 \pm 1.6$ & $11.3 \pm 0.7^{\mathrm{b}}$ & $12.7 \pm 1.0^{\mathrm{b}}$ & $15.9 \pm 1.3^{\mathrm{b}}$ & $<0.001$ \\
\hline Desmosterol $^{\mathrm{a}}$ & $158.5 \pm 13.6$ & $77.7 \pm 2.1^{b}$ & $79.7 \pm 2.4^{b}$ & $91.5 \pm 3.1^{b}$ & $<0.001$ \\
\hline Lathosterol $^{\mathrm{a}}$ & $66.3 \pm 5.7$ & $60.1 \pm 3.5$ & $77.2 \pm 6.4$ & $100.9 \pm 8.6^{c}$ & $<0.001$ \\
\hline Campesterol $^{\mathrm{a}}$ & $154.4 \pm 35.3$ & $344.9 \pm 18.9^{b}$ & $361.6 \pm 19.0^{\mathrm{b}}$ & $313.3 \pm 23.1^{b}$ & $<0.001$ \\
\hline Sitosterol $^{\mathrm{a}}$ & $140.2 \pm 40.4$ & $197.8 \pm 8.9$ & $204.1 \pm 11.8$ & $174.2 \pm 14.1$ & 0.068 \\
\hline Stigmasterol $^{\mathrm{a}}$ & $19.0 \pm 2.3$ & $28.7 \pm 1.4^{b}$ & $30.8 \pm 3.0^{\mathrm{b}}$ & $26.1 \pm 1.4$ & 0.006 \\
\hline Avenasterol $^{\mathrm{a}}$ & $24.3 \pm 4.1$ & $53.0 \pm 2.7^{\mathrm{b}}$ & $52.8 \pm 3.0^{b}$ & $46.1 \pm 3.0^{\mathrm{b}}$ & $<0.001$ \\
\hline Cholestanol $^{\mathrm{a}}$ & $170.1 \pm 8.3$ & $176.3 \pm 5.0$ & $180.5 \pm 8.6$ & $168.3 \pm 7.2$ & 0.628 \\
\hline
\end{tabular}

Mean \pm SE.

a $10^{2} \mu \mathrm{mol} / \mathrm{mmol}$ cholesterol.

b Significantly different from age $<1$ year.

c Significantly different from all other age groups. 
Weight was available from 34 girls and 55 boys, but height was measured only in 6 girls and 21 boys, so that body mass index was not calculated. Weight ranged from $6.2 \mathrm{~kg}$ to $64.0 \mathrm{~kg}$ in girls and from 4.4 to $76.7 \mathrm{~kg}$ in boys, respectively. The mean serum cholesterol concentration was $3.83 \pm 0.07 \mathrm{mmol} / \mathrm{l}$ in the whole study population ranging from 2.52 to $5.01 \mathrm{mmol} / \mathrm{l}$ in the girls and from 2.21 to $5.47 \mathrm{mmol} / \mathrm{l}$ in the boys, with no difference between the genders. The main reasons for the day-surgery were: corrections of umbilical or inguinal hernia or testis retention, removal of nevus or other skin, subcutaneous, cartilage, or bone lesions, all benign in histology, arthroscopy, different endoscopies, or circumcision.

The study population was divided into 5-year age groups 1-5 $(\mathrm{n}=37), 6-10(\mathrm{n}=24)$, and $11-15$ years $(\mathrm{n}=21)$ (Table 1$)$. Less than 1 -year old $(n=14)$ subjects were left as their own group because some of the serum non-cholesterol sterols behaved differently in the first year of life compared with the other age groups. According to power analysis based on previous data [2], with an $\alpha$ level of 0.05 (two-sided) and statistical power of 0.80 , the required minimal study population per age group should be 12 , so that each group fulfilled the criteria of optimal size. In the age group 1-5 years, the boys were heavier than the girls $(17.9 \pm 0.8 \mathrm{~kg}$ vs. $14.5 \pm 0.7 \mathrm{~kg}, p=0.008)$, but in the other age groups there was no gender difference in weight. Serum cholesterol concentration was higher in the girls than in the boys in the age group $<1$ year $(4.14 \pm 0.28 v s .3 .18 \pm 0.19 \mathrm{mmol} / 1, p=0.047)$, but in the other age groups neither serum cholesterol nor non-cholesterol sterols differed between the genders.

\subsection{Serum cholesterol and non-cholesterol sterols}

In the whole study population, serum cholesterol concentration increased by age $(r=0.247, p<0.01)$, and in the age group 6-10 years it was significantly higher than in the age group $<1$ year (Table 1). Of the non-cholesterol sterols, only lathosterol increased by age $(r=0.460, p<0.001)$, and it was higher in the oldest age group compared with the three younger age groups (Table 1). Lathosterol was also the only non-cholesterol sterol correlating with weight $(r=0.557$, $p<0.001$ ).

Cholestenol and desmosterol were highest in the age group $<1$ year, after which they were similar between the other age groups (Table 1). Squalene was higher in the age group $<1$ year compared with the age groups 1-5 and 6-10 years.

Serum campesterol, stigmasterol, and avenasterol were lowest in the age group $<1$ year (Table 1 ). Sitosterol, too, tended to be lowest in the age group $<1$ year $(p=0.068)$. The lowest plant sterol values were observed in children less than six months of age, and campesterol and avenasterol correlated with age in the age group $<1$ year (campesterol $(\mathrm{r}=0.599, p<0.05$, and avenasterol $\mathrm{r}=0.787, p<0.001)$. In the three older age groups, the plant sterol ratios did not differ between the groups. Cholestanol was similar between all age groups.

The ratios of cholestenol and lathosterol to cholestanol were highest in the age group 11-15 years, shown for lathosterol/cholestanol in Fig. 1. Lathosterol/desmosterol ratio was lowest in the age group $<1$ year, but from the age of 6 years the ratio was no more changing. Campesterol/cholestanol ratio was lowest in the age group $<1$ year, after which it did not differ between the groups.

\subsection{Interrelations of cholesterol precursors, plant sterols, and cholestanol}

In the whole study population, lathosterol correlated with cholestenol, and the relationship was significant in all age groups (Table 2). Squalene correlated with the other precursors only in the age group 6-10 years, shown for squalene-lathosterol in Table 2. Desmosterol correlated with lathosterol only in the oldest age group $(r=0.740$, $p<0.001)$.

Campesterol, sitosterol, and avenasterol were interrelated in the whole study population and in all age groups (Table 2) except for
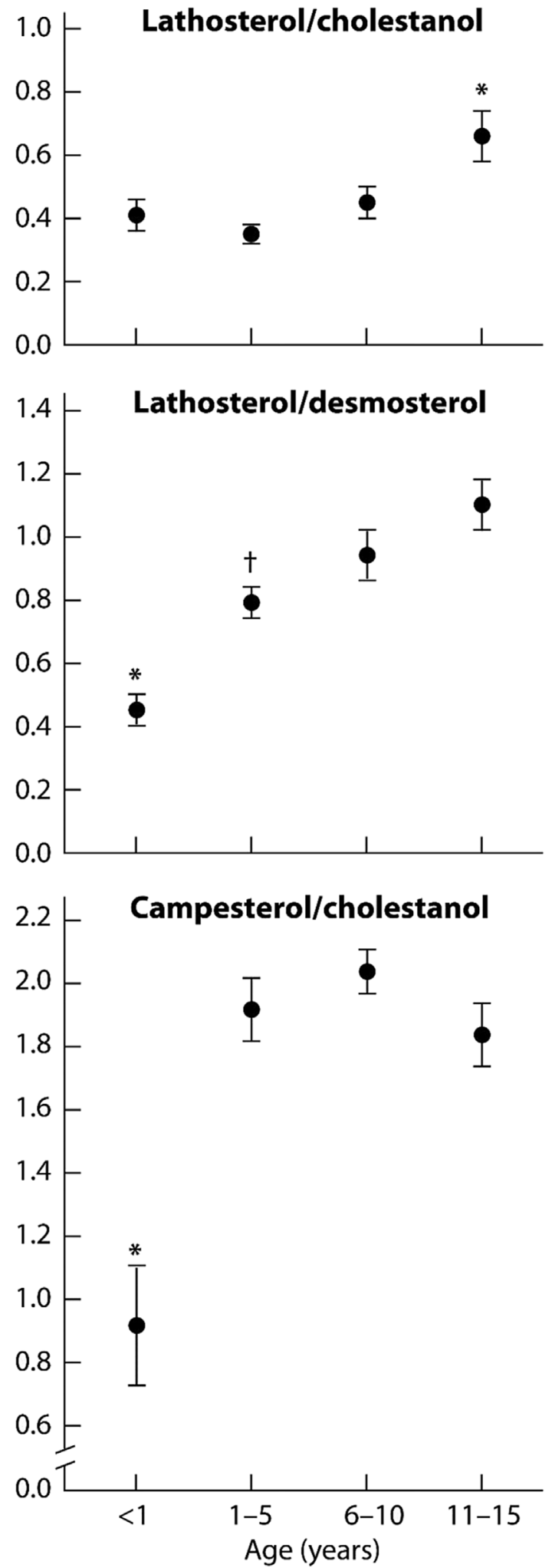

Fig. 1. Ratios of serum lathosterol/cholestanol, lathosterol/desmosterol, and campesterol/cholestanol (all $\mu \mathrm{g} / \mu \mathrm{g}$ ) in healthy children and adolescents at the age of $<1$ year $(n=14), 1-5$ years $(n=37), 6-10$ years $(n=24)$, and $11-15$ years $(n=21)$. Mean \pm SE. Analysis of variance, $p<0.001$, *from all other age groups, †from the age group 11-15 years.

sitosterol-avenasterol in the age group $<1$ year (data not shown). Cholestanol correlated with campesterol, sitosterol, and avenasterol in the whole study population and in all other age groups except the age 
Table 2

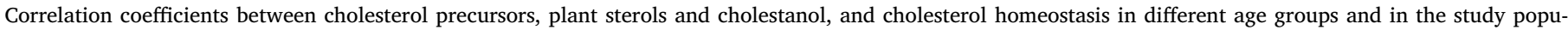
lation.

\begin{tabular}{|c|c|c|c|c|c|}
\hline Variables & Age $<1$ year $n=14$ & Age $1-5$ years $n=37$ & Age $6-10$ years $n=24$ & Age $11-15$ years $n=21$ & Total $\mathrm{n}=96$ \\
\hline \multicolumn{6}{|l|}{ Cholesterol precursors } \\
\hline Squalene-lathosterol & 0.285 & 0.151 & $0.661^{\mathrm{c}}$ & 0.158 & 0.174 \\
\hline Cholestenol-lathosterol & $0.785^{c}$ & $0.563^{\mathrm{c}}$ & $0.923^{\mathrm{c}}$ & $0.930^{\mathrm{c}}$ & $0.774^{\mathrm{c}}$ \\
\hline \multicolumn{6}{|c|}{ Plant sterols and cholestanol } \\
\hline Campesterol-sitosterol & $0.717^{\mathrm{b}}$ & $0.909^{c}$ & $0.694^{\mathrm{c}}$ & $0.899^{c}$ & $0.826^{\mathrm{c}}$ \\
\hline Campesterol-avenasterol & $0.823^{c}$ & $0.726^{\mathrm{c}}$ & $0.775^{\mathrm{c}}$ & $0.728^{\mathrm{c}}$ & $0.831^{\mathrm{c}}$ \\
\hline Cholestanol-campesterol & 0.196 & $0.649^{c}$ & $0.746^{\mathrm{c}}$ & $0.773^{\mathrm{c}}$ & $0.677^{c}$ \\
\hline \multicolumn{6}{|l|}{ Cholesterol homeostasis } \\
\hline Lathosterol-campesterol & -0.285 & -0.286 & $-0.449^{\mathrm{a}}$ & $-0.779^{c}$ & $-0.351^{\mathrm{c}}$ \\
\hline Lathosterol-cholestanol & -0.303 & $-0.417^{\mathrm{a}}$ & -0.363 & $-0.740^{c}$ & $-0.454^{c}$ \\
\hline
\end{tabular}

$\mu \mathrm{g} / \mu \mathrm{g}$

${ }^{\mathrm{a}} p<0.05$.

b $p<0.01$.

c $p<0.001$.

group $<1$ year shown for campesterol-cholestanol in Table 2. Stigmasterol correlated weakly with the other plant sterols and cholestanol in the whole study population (r-values from 0.212 to 0.405 , $p<0.05$ ), but not in the different age groups (data not shown).

\subsection{Cholesterol homeostasis evaluated with non-cholesterol sterols}

In the age group $<1$ year, cholesterol precursors did not associate with plant sterols or cholestanol shown for lathosterol-campesterol and lathosterol-cholestanol in Table 2. From the age group 1-5 years, lathosterol-cholestanol or lathosterol-campesterol were inversely interrelated. Stigmasterol did not associate with any of the cholesterol precursors.

\section{Discussion}

The new observations in the present study were as follows: serum non-cholesterol sterols had different individual profiles by age in childhood and adolescence from 0 to 15 years of age. These differences could not be explained by age-related changes in the concentrations of serum cholesterol and transporter lipoproteins, because the non-cholesterol sterols were adjusted to cholesterol. There were no gender differences in the non-cholesterol sterols in these age groups. Squalene, cholestenol and desmosterol were higher in the age group $<1$ year compared with the older age groups, whereas lathosterol was highest in the age group 11-15 years. Plant sterols were low during the first year of life and especially during the first six months of age. Cholestanol was not related to age. Second, cholesterol homeostasis was not intact until 1 year of age. Third, from 1 to 10 years of age, cholesterol absorption prevailed cholesterol synthesis.

Of the age-related differences in serum non-cholesterol sterols, we first deal with the cholesterol precursors. After birth, the markedly elevated serum desmosterol values decreased during the first year of life, and after 1 year of age they were similar between the other age groups. Since breast milk and infant formulas contain plenty of desmosterol, it is possible that the high serum desmosterol levels primarily reflected their increased dietary intake rather than cholesterol synthesis. Breast milk contains desmosterol about $1 \mathrm{mg} / 100 \mathrm{ml}(0.03 \mathrm{mmol} /$ 1) depending on the state of lactation, so that at the age of 5 months its dietary intake can be up to $5-10 \mathrm{mg} / \mathrm{d}[13,22-24]$. The concept that serum desmosterol is primarily diet-derived after birth is also supported by the finding that in this age group desmosterol did not correlate with any of the other cholesterol synthesis markers. On the other hand, the elevated squalene and cholestenol levels $<1$ year of age suggested that cholesterol synthesis was activated in this age group.

Serum lathosterol level remained practically unchanged after birth throughout the childhood until it increased in adolescence. This finding resembles the results of an earlier study in 12-, 15-, and 18-year old boys, in whom serum lathosterol increased gradually after 12 years of age [14]. Accordingly, the present results suggest that during the first year of life, and from the age of 11 years, cholesterol synthesis is activated. However, during the first year of life, only the 'early' precursors squalene and cholestenol are elevated in serum, whereas in the adolescence serum lathosterol, the 'late' precursor is elevated. It remains open why the cholesterol synthesis pathway is differently activated in different ages. However, the lathosterol/desmosterol ratio showed that from 6 years of age cholesterol synthesis was practically similarly divided between the unsaturated Bloch and the saturated KandutschRussel pathways.

Plant sterols are not synthesized in the human body, and they are diet-derived. Breast milk contains very little plant sterols, about 0.1 $\mathrm{mg} / 100 \mathrm{ml}(0.003 \mathrm{mmol} / \mathrm{l})$ [13], whereas infant formulas contain more, about $4 \mathrm{mg} / 100 \mathrm{ml}(0.1 \mathrm{mmol} / \mathrm{l})$ [24]. Thus, the low campesterol/cholestanol ratio, which reflects the plant sterol intake [21], suggested that the low serum plant sterol levels reflected primarily their low intake. Accordingly, in this age group they cannot be considered as cholesterol absorption markers.

Serum cholesterol precursors, plant sterols, and cholestanol are biomarkers of whole-body cholesterol synthesis and absorption efficiency validated in several adult populations [15-19]. To be certain that they operated as expected also in this child population, we interrelated the cholesterol precursors with each other in every age group as well as the absorption markers. In addition, cholesterol homeostasis was checked by relating the precursor sterols to the absorption markers in every age group. It seems evident that cholestenol and lathosterol were the most reliable biomarkers of cholesterol synthesis in every age group. Interestingly, only in the age group 6-10 years, squalene correlated with all other precursors. Serum squalene was originally reported as a marker of cholesterol synthesis especially in non-steadystate situations [25], but later on, it was not associated with cholesterol synthesis except in subjects with type 2 diabetes [19]. Its correlation with the other precursors, especially in the age group 6-10 yrs, probably reflects a burst in cholesterol synthesis during these years.

Serum plant sterols except stigmasterol were tightly interrelated. In the age group $<1$ year, they did not correlate with cholestanol, but from the age of 1 year, plant sterols and cholestanol were interrelated. Stigmasterol behaved differently, it associated only weakly with the other plant sterols and cholestanol in the whole study population. Stigmasterol was neither associated with any of the cholesterol precursors. Accordingly, even though being a plant sterol, stigmasterol cannot be considered as a marker of cholesterol absorption. Stigmasterol is the only plant sterol which in large concentrations, simulating human phytosterolemia, is able to interfere with cholesterol homeostasis. Thus, in adrenal cells of mice lacking ABCG5 and ABCG8 
receptors, it reduced cholesterol synthesis by inhibiting SREBP-2 processing, and it was able to activate the liver $\mathrm{X}$ receptor [26].

During the first year of life, there was no interaction between cholesterol precursors and cholestanol, suggesting that cholesterol homeostasis was not yet developed. From the age of 1 year, cholesterol homeostasis could be considered intact. According to Fig. 1, cholesterol absorption prevailed cholesterol synthesis from the age of 1 to the age of 10 years. Thus, the lathosterol/cholestanol ratios shown in Fig. 1 increased from the low levels in the 1-10 year old (0.35-0.45) to 0.66 in the age group 11-15 years. In fact, a similar lathosterol/cholestanol ratio was observed earlier in 12-year old boys in The Cardiovascular Risk in Young Finns Study, and the ratio still increased up to about 0.80 in the 18-year-old boys [14]. These previous results confirm our conclusion that in adolescence cholesterol synthesis prevails cholesterol absorption. Whether this childhood profile of cholesterol metabolism from 1 to 10 years of age with prevailing cholesterol absorption is proatherogenic warrants further investigation. However, the prevailing cholesterol absorption emphasizes to consider the importance of dietary cardiovascular risk factors even from early childhood.

\subsection{Limitations}

The study population was a consecutive cohort of $0-15$ year-old day-surgery patients, who had a minor operation, removal of a benign lesion, or endoscopy. The subjects had fasted overnight, and the blood sample was drawn in the morning before the procedure. Otherwise the subjects were considered healthy and they had followed their habitual home diet so that it is conceivable that their cholesterol metabolism was not interfered and they can be considered as a healthy study population in steady-state. The assessment of the validity of serum noncholesterol sterols had to be based on the information obtained from this study and based on whether cholesterol homeostasis was intact, and whether the synthesis markers as well as the absorption markers were interrelated. The absolute measurements of cholesterol metabolism would have been impossible to perform in small children and in a study population of this magnitude. Regarding the sample size, the number of subjects in the different age groups exceeded the demands of the power calculation. However, in the age group $<1$ years of age the number of subjects was rather low even though excessing the limit of power calculation, and somewhat gender-unbalanced, though statistically non-significant towards boys $v s$. girls. Moreover, a detailed dietary information or the breastfeeding status was not recorded, so that data should be interpreted with caution in this age group.

\subsection{Conclusions}

We have analysed cholesterol metabolism with serum non-cholesterol sterol ratios to cholesterol in 0-15 year-old children and adolescents without dyslipidemia. First, serum non-cholesterol sterols had different individual profiles by age. Of the cholesterol synthesis markers squalene and cholestenol were higher in the age group $<1$ year compared with the older age groups, whereas lathosterol was highest in the age group 11-15 years suggesting that cholesterol synthesis was activated in both age groups but with different biomarkers. Cholestanol was not related to age. In the age group $<1$ year of age high serum desmosterol and low plant sterols conceivably reflected their dietary intake and cannot be used as biomarkers. Second, cholesterol homeostasis was not intact until from 1 years of age. Third, from 1 to 10 years of age, cholesterol absorption prevailed cholesterol synthesis. This novel finding emphasizes the importance of dietary aspects related to cardiovascular risk even from early childhood.

\section{Conflicts of interest}

The authors declared they do not have anything to disclose regarding conflict of interest with respect to this manuscript.

\section{Author contributions}

All authors (HG, MK, AM, MJN, MP, and PS) were involved in the conception and design of this study. AM and MP participated in the acquisition of the clinical data. MK analysed the data, and HG, MK and PS performed the final statistical analyses and interpretation of the data. HG drafted and finalised the manuscript. MK, AM, MJN, MP, and PS critically reviewed and revised the manuscript. All authors have approved this final version to be submitted.

\section{Acknowledgements}

Ms Leena Kaipiainen is acknowledged for excellent technical assistance.

\section{References}

[1] J.J. Hamilton, A. Synnes, S.M. Innis, Plasma cholesterol and lathosterol levels in term infants in the early neonatal period, Pediatr. Res. 31 (1992) 396-400.

[2] A.F. Vuorio, T.A. Miettinen, H. Turtola, H. Oksanen, H. Gylling, Cholesterol metabolism in normal and heterozygous familial hypercholesterolemic newborns, J. Lab. Clin. Med. 140 (2002) 35-42.

[3] A. Correani, S. Visentin, E. Cosmi, E. Ponchia, S. D'Aronco, et al., The maternal-fetal gradient of free and esterified phytosterols at the time of delivery in humans, Clin. Nutr. (2017) (Epub ahead of print).

[4] H.E. Miettinen, K. Rönö, S.B. Koivusalo, J.G. Eriksson, H. Gylling, Effect of gestational diabetes mellitus on newborn cholesterol metabolism, Atherosclerosis 275 (2018) 346-351.

[5] M.J.T. Kallio, L. Salmenperä, M.A. Siimes, J. Perheentupa, T.A. Miettinen, Exclusive breast-feeding and weaning: effect on serum cholesterol and lipoprotein concentrations in infants during the first year of life, Pediatrics 89 (1992) 663-666.

[6] K.V.K. Porkka, J.S.A. Viikari, H.K. Åkerblom, Tracking of serum HDL-cholesterol and other lipids in children and adolescents: the cardiovascular risk in young Finns study, Prev. Med. 20 (1991) 713-724.

[7] A. Wiegman, S.S. Gidding, G.F. Watts, M.J. Chapman, H.N. Ginsberg, et al., For the European Atherosclerosis Society Consensus Panel Familial hypercholesterolaemia in children and adolescents: gaining decades of life by optimizing detection and treatment, Eur. Heart J. 36 (2015) 2425-2437.

[8] T.A. Miettinen, H. Gylling, T. Strandberg, S. Sarna, Baseline serum cholestanol as predictor of recurrent coronary events in subgroup of Scandinavian simvastatin survival study. Finnish 4S investigators, Br. Med. J. 316 (1998) 1127-1130.

[9] D. Teupser, R. Baber, U. Ceglarek, M. Scholz, T. Illig, et al., Genetic regulation of serum phytosterol levels and risk of coronary artery disease, Circ Cardiovasc Genet 3 (2010) 331-339.

[10] G. Silbernagel, M.J. Chapman, B. Genser, M.E. Kleber, G. Fauler, et al., High intestinal cholesterol absorption is associated with cardiovascular disease and risk alleles in ABCG8 and ABO.Evidence from the LURIC and YTS cohorts and from a meta-analysis, J. Am. Coll. Cardiol. 62 (2013) 291-299.

[11] The Myocardial Infarction Genetics Consortium Investigators, Inactivating mutations in NPC1L1 and protection from coronary heart disease, N. Engl. J. Med. 371 (2014) 2072-2082.

[12] A. Tammi, T. Rönnemaa, L. Valsta, L. Seppänen, L. Rask-Nissilä, et al., Dietary plant sterols alter the serum plant sterol concentration but not the cholesterol precursor sterol concentrations in young children (the STRIP study), J. Nutr. 131 (2001) 1942-1945.

[13] K. Laitinen, E. Isolauri, L. Kaipiainen, H. Gylling, T.A. Miettinen, Plant stanol ester spreads as components of a balanced diet for pregnant and breast-feeding women; evaluation of clinical safety, Br. J. Nutr. 101 (2009) 1797-1804.

[14] T.A. Miettinen, H. Gylling, J. Viikari, T. Lehtimäki, O.T. Raitakari, Synthesis and absorption of cholesterol in Finnish boys by serum non-cholesterol sterols. The cardiovascular risk in young Finns study, Atherosclerosis 200 (2008) 177-183.

[15] I. Björkhem, T. Miettinen, E. Reihnér, S. Ewerth, B. Angelin, et al, Correlation between serum levels of some cholesterol precursors and activity of HMG-CoA reductase in human liver, J. Lipid Res. 28 (1987) 1137-1143.

[16] T.A. Miettinen, R.S. Tilvis, Y.A. Kesäniemi, Serum cholestanol and plant sterol levels in relation to cholesterol metabolism in middle-aged men, Metabolism 38 (1989) 136-140.

[17] T.A. Miettinen, R.S. Tilvis, Y.A. Kesäniemi, Serum plant sterols and cholesterol precursors reflect cholesterol absorption and synthesis in volunteers of a randomly selected male population, Am. J. Epidemiol. 131 (1990) 20-31.

[18] J.C. Cohen, A. Pertsemlidis, S. Fahmi, S. Esmail, G.L. Vega, et al., Multiple rare variants in NPC1L1 associated with reduced sterol absorption and plasma lowdensity lipoprotein levels, Proc. Natl. Acad. Sci. U.S.A. 103 (2006) 1810-1815.

[19] P. Simonen, H. Gylling, T.A. Miettinen, The validity of serum squalene and noncholesterol sterols as surrogate markers of cholesterol synthesis and absorption in type 2 diabetes, Atherosclerosis 197 (2008) 883-888.

[20] T.A. Miettinen, Cholesterol metabolism during ketoconazole treatment in man, J. Lipid Res. 29 (1988) 43-51.

[21] X. Lin, S.B. Racette, L. Ma, M. Wallendorf, C.A. Spearie, et al., Plasma biomarker of dietary phytosterol intake, PLoS One 10 (2) (2015) e0116912. 
[22] R.M. Clark, M.B. Fey, R.G. Jensen, D.W. Hill, Desmosterol in human milk, Lipids 18 (1983) 264-266.

[23] M.J. Kallio, M.A. Siimes, J. Perheentupa, L. Salmenperä, T.A. Miettinen, Cholesterol and its precursors in human milk during prolonged exclusive breast-feeding, Am. J. Clin. Nutr. 4 (1989) 782-785.

[24] L. Claumarchirant, E. Matencio, L.M. Sanchez-Siles, A. Alegría, M.J. Lagarda, Sterol composition in infant formulas and estimated intake, J. Agric. Food Chem. 63 (2015) 7245-7251.

[25] D.S. Goodman, Squalene in human and rat blood plasma, J. Clin. Invest. 43 (1964) 1480-1485.

[26] C. Yang, L. Yu, W. Li, F. Xu, J.C. Cohen, et al., Disruption of cholesterol homeostasis by plant sterols, J. Clin. Invest. 114 (2004) 813-822. 